Reprod. Nutr. Dévelop., 1988, 28 Suppl. n 1, 151-152

\title{
Mesure du transit digestif chez le veau ruminant : comparaison et validation de modèles d'ajustement des cinétiques de passage
}

\author{
J. P. LALLES, E. DELVAL, C. PONCET
}

Station de Recherches sur la Nutrition des Herbivores, Unité d'Etude de la Dynamique de la Digestion.

IN.R.A., Theix, 63122 Ceyrat, France.

Summary. Three models of marker passage analysis were compared. None of them was found to be superior in fitting the data. The 3 models provided similar estimates of the mean retention time of hay in the whole digestive tract and in the rumen of calves. Discrepancies between models for other compartments are discussed in relation to sampling sites.

La modélisation des cinétiques de passage des aliments dans le tube digestif (TD) permet d'estimer leur temps de séjour moyen (TSM) dans l'ensemble (TSMT) du TD et dans ses compartiments. Nous avons comparé 3 modèles constitués de 2 compartiments de mélange et d'un compartiment tubulaire: A (Grovum et Williams, 1973), B (Ellis et al., 1979), C (Dhanoa et al., 1985), et testé la validité de l'interprétation physiologique de leurs paramètres en appliquant ces modèles au niveau fécal et duodénal.

Matériel et méthodes. Des cinétiques de passage duodénal $(n=13)$ et d'excrétion fécale $(n=13)$ de foin marqué (Ytterbium 169) ont été obtenues aux âges de 4 et 5 mois, chez 7 veaux sevrés recevant une ration riche en aliment concentré. Le foin de fétuque, distribué en 2 repas/j, représentait $20 \%$ de la matière sèche ingérée. Les fèces et le contenu duodénal ont été collectés (20 prélèvements par cinétique) respectivement pendant 5 et 3 jours après la distribution d'une dose de foin marqué. Les comparaisons des paramètres de transit entre modèles et entre lieux de prélèvement ont été effectuées par régression linéaire.

Résultats et discussion. La qualité d'ajustement des courbes fécales et duodénales est similaire pour les 3 modèles (tabl. 1), alors que Dhanoa et al. (1985) ont obtenu les meilleurs résultats, au niveau fécal, par le modèle $C$. L'estimation du TSMT est identique entre modèles et non différente de celle obtenue $(41,6 \pm 6,0 \mathrm{~h})$ algébriquement (Faichney, 1975). La modélisation, assimilant l'excrétion fécale à un phénomène continu, ne biaise donc pas l'estimation du TSMT. L'ajustement des courbes fécales montre que les 3 modèles conduisent à des estimations identiques du TSM dans le compartiment 1 (TSM1), assimilé au rumen dans les 3 modèles. Le modèle $C$ donne des estimations de TSM2 et TSM3 différentes de celles obtenues par les 2 autres. Les différences entre les modèles $A$ et $C$ sont difficiles à expliquer car ils sont de conception voisine avec 2 compartiments de mélange distincts (rumen, caecum- 
côlon), à taux de renouvellement constants. L'application du modèle B pourrait conduire à des résultats différents car dans ce modèle, les 2 compartiments de mélange sont localisés dans le rumen, le taux de renouvellement du contenu de l'un, identifié au mécanisme de réduction de taille des particules, dépendant du temps. Les différences de conception des modèles et d'identification de leurs paramètres peuvent être révélées par l'analyse des cinétiques duodénales.

Pour les 3 modèles, l'estimation duodénale de TSM1 est supérieure à l'estimation fécale. Lors de l'analyse des cinétiques fécales, une partie du TSM1 est reportée sur le TSM des autres compartiments, ce qui met en doute l'utilisation des cinétiques fécales pour estimer TSM1. Si le premier ou les 2 compartiments de mélange étaient associés au rumen (modèle $B$ ), le lieu de prélèvement ne devrait pas modifier l'estimation de TSM1 et de TSM2, hypothèse seulement vérifiée pour les TSM1 obtenus au niveau duodénal et fécal par le modèle $\mathrm{C}$. Le compartiment 2 du modèle $A$ est assimilé au caecum-côlon dans le cas des cinétiques fécales (Grovum et Williams, 1973) et à la caillette dans le cas des cinétiques duodénales (Faichney et Griffiths, 1978). L'estimation de TSM2 doit alors dépendre du lieu de prélèvement, ce qui est observé.

En conclusion, aucun des 3 modèles étudiés n'a permis de déterminer la répartition réelle du TSM du foin entre les différents compartiments du TD. Des mesures directes sont nécessaires pour préciser la nature et la localisation des phénomènes associés aux compartiments des modèles.

TABL. 1. - Influence du modèle et du lieu de prélèvement sur le temps de séjour moyen du foin dans lensemble du tube digestif (TSMT), les estomacs (TSME), le premier compartiment de mélange (TSM1), le deuxième compartiment de mélange (TSM2) et les compartiments tubulaires (TSM3) (moyenne et ET).

\begin{tabular}{lcccc}
\hline $\begin{array}{l}\text { Lieu de } \\
\text { prélèvement }\end{array}$ & Variable & $\begin{array}{c}\text { Modèle A (Grown } \\
\text { et Williams, 1973) }\end{array}$ & $\begin{array}{c}\text { Modèle B (Ellis } \\
\text { et al. 1979) }\end{array}$ & $\begin{array}{c}\text { Modèle C (Dhanoa } \\
\text { et al. 1985) }\end{array}$ \\
\hline \multirow{4}{*}{$\begin{array}{c}\text { Fèces } \\
(\mathrm{n}=13)\end{array}$} & ETR (\% moyenne) & $12,9(5,1)$ & $13,0(5,5)$ & $12,4(5,5)$ \\
& TSMT (h) & $41,0(5,0)$ & $41,5(5,8)$ & $41,1(5,0)$ \\
& TSM1 (h) & $22,4(5,6) \mathrm{d}$ & $23,8(7,2) \mathrm{d}$ & $24,4(5,1)$ \\
& TSM2 (h) & $6,5(2,0) \mathrm{a}, \mathrm{f}$ & $7,2(3,0) \mathrm{a}, \mathrm{f}$ & $3,7(0,7) \mathrm{b}, \mathrm{f}$ \\
& TSM3 (h) & $11,3(2,0) \mathrm{a}$ & $10,5(2,9) \mathrm{a}$ & $13,1(1,7) \mathrm{b}$ \\
\hline \multirow{4}{*}{$\begin{array}{c}\text { Duodénum } \\
\text { (n=13) }\end{array}$} & ETR (\% moyenne) & $9,6(2,0)$ & $9,7(1,7)$ & $9,7(1,9)$ \\
& TSME (h) & $30,5(7,1) \mathrm{a}$ & $33,0(8,6) \mathrm{b}$ & $33,2(7,9) \mathrm{b}$ \\
& TSM1 (h) & $26,1(8,0) \mathrm{a}, \mathrm{e}$ & $29,4(9,4) \mathrm{b}, \mathrm{e}$ & $29,4(8,8) \mathrm{b}$ \\
& TSM2 (h) & $3,7(1,8) \mathrm{a}, \mathrm{g}$ & $3,4(1,7) \mathrm{a}, \mathrm{g}$ & $1,9(0,9) \mathrm{b}, \mathrm{g}$ \\
& TSM3 (h) & $0,6(0,7) \mathrm{a}$ & $0,2(0,5) \mathrm{a}$ & $1,9(0,8) \mathrm{b}$ \\
\hline
\end{tabular}

ETR : écart-type résiduel des ajustements (\% de la moyenne des concentrations en marqueur). Différences entre modèles $(a, b, c)$ et entre lieux pour TSM1 $(d, e)$ et TSM2 $(f, g) ; P<0,05$.

Dhanoa M., Siddons R., France J., Gale D., 1985. Br. J. Nutr., 53, 663-671.

Ellis W. C., Matis J. H., Lascano C., 1979. Fed. Proc., 38, 2702-2706.

Faichney G. J., 1975. Aust. J. agric. Res., 13, 319-327.

Faichney G. J., Griffiths G. A., 1978. Br. J. Nutr., 40, 71-82.

Grovum W. L., Williams V. J., 1973. Br. J. Nutr., 30, 231-240. 